\title{
Bioenergetic Analysis of FLAG Tagged-Subunit 8 of Saccharomyces cerevisiae Mitochondrial ATP Synthase
}

\author{
I MADE ARTIKA \\ Department of Biochemistry, Faculty of Mathematics and Natural Sciences, \\ Institut Pertanian Bogor, Darmaga Campus, Bogor16680, Indonesia \\ Phone/Fax.+62-251-8423267, E-mail: imart171@yahoo.com
}

\begin{abstract}
The majority of cellular energy in the form of adenosine triphosphate (ATP) is synthesized by the $\mathrm{F}_{1} \mathrm{~F}_{0}$-ATP synthase. The yeast mitochondrial $\mathrm{F}_{1} \mathrm{~F}_{0}-\mathrm{ATP}$ synthase is a multisubunit complex that contains at least 17 different subunits grouped into $\mathrm{F}_{1}$ and $\mathrm{F}_{0}$ sectors. Subunit 8 of yeast mitochondrial ATP synthase is a hydrophobic protein of 48 amino acids encoded by the mitochondrial ATP 8 gene. Subunit 8 has three distinct domains; an $\mathrm{N}$-terminal domain, a central hydrophobic domain and a C-terminal domain. FLAG tag addition to the C-terminus of subunit 8 and its variants has facilitated elucidation of subunit 8 's membrane topology. In order to analyze its detailed structure and function, a set of strains expressing FLAG tagged-subunit 8 and its variants were subjected to bioenergetic analysis at cellular and mitochondrial levels. Results obtained showed that the hydrophobic character of the central hydrophobic domain of subunit 8 is essential for functional coupling between $\mathrm{F}_{1}$ and $\mathrm{F}_{0}$ sectors, hence for mitochondrial ATP synthase function.
\end{abstract}

Key words: bioenergetics, ATP synthase, mitochondria, yeast

The majority of cellular energy in the form of adenosine triphosphate (ATP) is synthesized by the $\mathrm{F}_{1} \mathrm{~F}_{0}$-ATP synthase. Power for ATP synthesis is derived from an electrochemical proton gradient generated by an electron transport chain in an energy transducing membrane. This force drives rotation of membranous $\left(\mathrm{F}_{0}\right)$ motor components. Rotation of the components of the $\mathrm{F}_{0}$ motor is transmitted to the eccentric shaft subunit to elicit conformational changes in the catalytic site $\mathrm{F}_{1}$, leading to ATP synthesis (van Ballmos et al. 2009). In some organisms, the ATP synthase also works in the reverse direction by hydrolyzing ATP and generating an electrochemical proton gradient across a membrane to support locomotion or nutrient uptake (Hong and Pedersen 2008).

ATP synthase is an exceptionally complicated protein complex. The yeast mitochondrial ATP synthase is composed of at least 17 subunits grouped into two sectors, a membrane-extrinsic sector $\left(\mathrm{F}_{1}\right)$ and a membrane-embedded sector $\left(F_{0}\right)$. The two sectors are linked by protein stalks. The $\mathrm{F}_{1}$ sector is comprised of five subunits , , , and , and is coupled to proton flux through the $\mathrm{F}_{0}$ sector. The $\mathrm{F}_{0}$ sector spans the membrane and is composed of subunits b, OSCP, $d$, $\mathrm{e}, \mathrm{f}, \mathrm{g}, \mathrm{h}, \mathrm{i} / \mathrm{j}, \mathrm{k}$ which are encoded by nuclear genes, and subunits 6,8 , and 9 , which are encoded by mitochondrial genes (Stephens et al. 2003). In the inner mitochondrial membrane the ATP synthase complex can form a dimer (Fronzes et al.2006).

The subunit 8 of yeast mitochondrial ATP synthase is essential for oxidative phosphorylation (Macreadie et al. 1983). It has three distinct domains; an N-terminal domain, a central hydrophobic domain (CHD) and a C-terminal domain (Devenish et al. 1992). The topology of subunit 8, which is determined by introduced unique cysteine residues, indicates that its $\mathrm{N}$-terminus is located in the intermembrane space of mitochondria whereas the C-terminus is located within the mitochondrial matrix (Stephens et al. 2000). Further analysis employing cysteine-scanning mutagenesis showed that the first 14 and the last 13 amino acids were extrinsic to the lipid bilayer, indicating the existence of a 21 amino acid transmembrane spanning region (Stephens et al. 2003). It is essential for subunit 8 to maintain transmembrane topology for functioning (Artika 2009).

As a mitochondrially encoded protein, subunit 8 is transcribed and translated entirely within the organelle. Subunit 8 is not present in prokaryotes which means that prokaryotic ATP synthase can naturally function without the presence of subunit 8 (Artika 2007). The immediate question therefore is to resolve the detailed structure and roles of this subunit in the enzyme complex. Several lines of evidence suggested that subunit 8 is part of the stator stalk in the yeast mitochondrial ATP synthase. No amino acid of subunit 8 directly participates in either ATP synthesis/hydrolysis or proton pumping, suggesting that subunit 8 is a structural component of the mitochondrial ATP synthase complex (Stephens et al.2003).

In order to elucidate its detailed structure, function, and membrane topology, an allotropic expression system for subunit 8 has been developed (Gearing et al. 1985). This system has been applied to study various aspects of subunit 8 molecular biology and has also been successfully used to express FLAG tagged-subunit 8 (Artika 2006) and FLAG tagged-subunit 8 variants (Artika 2007). The main purpose of the FLAG tag addition to subunit 8 was to enable detection of subunit 8 protein by means of an anti-FLAG tag monoclonal antibody.

The FLAG tag system has been found to be useful in the studies of abundance, cellular location, posttranslational modifications, protein-protein interactions, and purification of particular tagged-protein (Kolodziej and Young 1991). The practicability of the FLAG tag system relies on the specificity of the binding of the anti-FLAG antibody to the FLAG fusion proteins (Schafer and Braun 1995). The small size of the FLAG epitope is proposed as having a minimal effect on protein conformation thereby reducing the possibility of disrupting the function of any tagged-protein. FLAG tagged-subunit 8 and its variants have been employed to study structure, function, and topology of this subunit (Artika 2007, 2009). The incorporation of FLAG epitope 
tag on the wild-type subunit 8 has been shown not to cause functional impairment on mitochondrial ATP synthase (Artika 2010). In the present study, strains expressing FLAG tagged-subunit 8 variants were subjected to cellular and mitochondrial respiration assays to analyze detailed subunit 8 's structure and function.

\section{MATERIALS AND METHODS}

Materials. Saccharomyces cerevisiae strain M31 [atp8, mit his6, ade1] a collection strain of the Department of Biochemistry and Molecular Biology, Monash University, has previously been described (Nagley et al. 1988). Strains FTC2, DF66, DF68, and DF71 have been described (Artika 2007). Strain FTC2 is strain M31 expressing FLAG-taggedsubunit 8 gene fused with a mitochondrial signal peptide. Strains DF66 is strain M31 expressing FLAG-taggedsubunit 8 variant (G16D, F17D) gene fused with a mitochondrial signal peptide. Strain DF68 is strain M31 expressing FLAG-tagged-subunit 8 variant (L23D, L24D) gene fused with a mitochondrial signal peptide. Strain DF71 is strain M31 expressing FLAG-tagged-subunit 8 variant (Q29R, F30R) gene fused with a mitochondrial signal peptide.

Isolation of Mitochondria. Isolation of mitochondria for bioenergetic analysis was carried out as described by Law et al. (1995). Unless otherwise stated, centrifugation steps were carried out using Sorvall SS34 rotor. Yeast cells were grown in 2 L flasks containing $450 \mathrm{~mL}$ Saccharomyces salt medium $[1 \%(\mathrm{w} / \mathrm{v})$ yeast extract, $0.12 \%(\mathrm{w} / \mathrm{v})$ $\left(\mathrm{NH}_{4}\right)_{2} \mathrm{SO}_{4}, \quad 0.1 \%$ (w/v) $\mathrm{KH}_{2} \mathrm{SO}_{4}, 0.01 \%$ (w/v) $\mathrm{CaCl}_{2}$, $0.0005 \%$ (w/v) $\mathrm{FeCl}_{3}, 0.07 \%$ (w/v) $\mathrm{MgCl}_{2}, 0.05 \%$ (w/v) $\mathrm{NaCl}$ ] supplemented with $2 \%(\mathrm{v} / \mathrm{v})$ ethanol until early midlogarithmic phase. After harvesting the cells by centrifugation using Sorvall RC3 rotor (4 $500 \mathrm{rpm}$; $5 \mathrm{~min}$ ), the cells were washed twice with ice cold distilled water containing $0.1 \%(\mathrm{w} / \mathrm{v})$ galactose. The cells were then resuspended in buffer 1 (0.1 M Tris-hydrochloric acid, $0.5 \mathrm{M}$ 2-mercaptoethanol, $\mathrm{pH} 9.3$ ) and incubated for $10 \mathrm{~min}$ at $30^{\circ} \mathrm{C}$. About $20 \mathrm{~mL}$ buffer $1 \mathrm{~g}^{-1}$ dry weight of cell was used. In order to remove the 2-mercaptoethanol, which may inhibit the enzymatic digestion step, the pellet was washed three times by resuspending the pellet in ice cold buffer 2 (10 $\mathrm{mM}$ Tris-hydrochloric acid, $0.5 \mathrm{M} \mathrm{KCl}, \mathrm{pH}$ 7.0) followed by centrifugation. About $50 \mathrm{~mL}$ buffer 2 was used per g cell. The cell walls were then digested by incubating the pellet in buffer 3 (10 mM citrate, $10 \mathrm{mM}$ disodium phosphate, $1.35 \mathrm{M}$ sorbitol, $1 \mathrm{mM}$ EGTA, pH 5.8) containing $1.0 \mathrm{mg} \cdot \mathrm{mL}^{-1}$ of zymolyase $20 \mathrm{~T}$ at $30^{\circ} \mathrm{C}$. The status of the digestion process was followed by measuring the optical density (OD) ${ }_{650 \mathrm{~nm}}$ of the suspension. The digestion was stopped when the optical density of the suspension reduced to $80 \%$. Following centrifugation (10 $000 \mathrm{rpm}$; $10 \mathrm{~min}$ ) the pellet was washed twice by resuspending the pellet in ice cold buffer $4(10 \mathrm{mM}$ Tris-maleate, $0.75 \mathrm{M}$ mannitol, $0.4 \mathrm{M}$ sorbitol, $2 \mathrm{mM}$ EGTA, $0.1 \%(\mathrm{w} / \mathrm{v})$ bovine serum albumin, $\mathrm{pH} 6.8)$ at $4{ }^{\circ} \mathrm{C}$ followed by centrifugation (10000 rpm; $10 \mathrm{~min}$ ). The pellet was then resuspended in buffer $5(10 \mathrm{mM}$ Tris-maleate, $0.6 \mathrm{M}$ mannitol, $2 \mathrm{mM}$ EGTA, $0.2 \%(\mathrm{w} / \mathrm{v})$ bovine serum albumin,
$0.5 \mathrm{mM}$ phosphate, $\mathrm{pH}$ 6.8). About $10 \mathrm{~mL}$ buffer 5 was used per $g$ dry cell weight. To release mitochondria from the spheroplast, the suspension was homogenized at high speed for $6 \times 5 \mathrm{sec}$. The suspension was diluted 4 times with buffer 5 followed by low speed centrifugation at $2000 \mathrm{rpm}$ for 20 min. The supernatant was decanted and centrifuged at high speed (12 $000 \mathrm{rpm} ; 10 \mathrm{~min}$ ) to recover the mitochondria. The mitochondrial pellet was washed twice by firstly resuspending the pellet in buffer $6(10 \mathrm{mM}$ Tris-maleate, 0.6 M mannitol, 2 mM EGTA, $0.5 \mathrm{mM}$ phosphate, $\mathrm{pH} 6.8$ ) followed by low speed centrifugation as before. Secondly, the supernatant was decanted and centrifuged at high speed (12 $500 \mathrm{rpm} ; 10 \mathrm{~min}$ ). Finally the mitochondria were resuspended in a small volume of buffer 6 prior to determination of protein concentration.

Cellular Respiration Assay. Cellular bioenergetic analysis was carried out by measuring the whole cell respiration as described by Law et al. (1995). Yeast cells were grown at $23^{\circ} \mathrm{C}$ on Saccharomyces salt medium [1\% $(\mathrm{w} / \mathrm{v})$ yeast extract, $0.12 \%(\mathrm{w} / \mathrm{v})\left(\mathrm{NH}_{4}\right)_{2} \mathrm{SO}_{4}, 0.1 \%(\mathrm{w} / \mathrm{v})$ $\mathrm{KH}_{2} \mathrm{SO}_{4}, 0.01 \%$ (w/v) $\mathrm{CaCl}_{2}, 0.0005 \%$ (w/v) $\mathrm{FeCl}_{3}, 0.07 \%$ (w/v) $\mathrm{MgCl}_{2}, 0.05 \%$ (w/v) $\mathrm{NaCl}$ ] plus $2 \%$ (v/v) ethanol, until they reached their mid-logarithmic growth phase. The cells were then harvested by centrifugation, washed twice in distilled water and then resuspended at $\mathrm{OD}_{550 \mathrm{~nm}}=30$. The cellular respiration rate was determined by measuring the oxygen consumption rate using the Clarke electrode (Gilson). The oxygraph was calibrated so that the amount of oxygen in $\mathrm{O}_{2}$-saturated distilled water in the chamber represented $100 \%$ and in the presence of sodium dithionate in the chamber represented $0 \%$ oxygen concentration. To the chamber was then added 300 L respiration buffer (Saccharomyces salt medium without ethanol). Following the addition of $1.0 \quad \mathrm{~L}$ of absolute ethanol, $10 \quad 1$ of cell suspension was added to the chamber. The rate of oxygen consumption, $\mathrm{J}_{0}$ (nmol $\mathrm{O} \mathrm{min}^{-1} \mathrm{mg}$ dry-cell-weight ${ }^{-1}$ ) was measured. The oxygen consumption rate measured in this state represents the physiological functioning of the cells. To measure the respiration rate in the absence of oxidative phosphorylation, $\mathrm{J}_{0}{ }^{\mathrm{TET}}$ (nmol O $\mathrm{min}^{-1} \mathrm{mg}$ dry-cell-weight ${ }^{-1}$ ), triethyltin (TET) was added to the chamber to a final concentration of $80 \mathrm{M}$. From these measurements, the respiratory control ratio (RCR), $\mathrm{J}_{0} / \mathrm{J}_{0}{ }^{\mathrm{TET}}$, was calculated. To determine the uncoupled (maximal) respiratory rate, carbonylcyanide $m$-chlorophenylhydrazone (CCCP) was added to the oxygraph chamber to a final concentration of 5 $\mathrm{mM}$. The maximal respiration rate, $\mathrm{J}_{0}{ }^{\mathrm{CCCP}}\left(\mathrm{nmol} \mathrm{O} \mathrm{min}^{-1} \mathrm{mg}\right.$ dry-cell-weight ${ }^{-1}$ ) was then measured. From these measurements, the maximum respiratory capacity (MRC), $\mathrm{J}_{0}{ }^{\mathrm{CCPP}} / \mathrm{J}_{0}{ }^{\mathrm{TET}}$, was calculated.

Mitochondrial Respiration Assay. The mitochondrial respiration rate was measured in the same oxygraph chamber used for determination of the cellular respiration rate. To the chamber 300 L of respiratory substrate containing $100 \mathrm{~g}$ available protein of mitochondria was added. The state 4 respiration [state $4(\mathrm{a})$ ] (nmol $\mathrm{O} \mathrm{min}^{-1} \mathrm{mg}$ mitochondrial protein $^{-1}$ ) was then measured. In order to induce state 3 
respiration, ADP was added to a final concentration of 1.0 $\mathrm{mM}$. The state 3 respiration ( $\mathrm{nmol} \mathrm{O} \mathrm{min}^{-1} \mathrm{mg}$ mitochondrial protein $^{-1}$ ) was then measured. From this measurement, the respiratory control ratio (RCR) was calculated. To measure the maximal respiratory capacity, the chamber was washed several times with distilled water. A fresh portion of $300 \mathrm{~L}$ respiratory buffer containing $100 \mathrm{~g}$ available protein of mitochondria was then added. The state 4 [state 4(b)] respiration (nmol O $\mathrm{min}^{-1} \mathrm{mg}$ mitochondrial protein ${ }^{-1}$ ) was measured as before and then CCCP was added to a final concentration of 13.3 $\mathrm{M}$ and the maximal respiration rate (nmol O min $^{-1} \mathrm{mg}$ mitochondrial protein ${ }^{-1}$ ) was measured. From these measurements the maximal respiratory capacity (MRC) was calculated.

\section{RESULTS}

Cellular Respiration Parameters of Strains Expressing Wild-Type and FLAG Tagged-subunit 8 Variants. The cellular respiration parameters of strains FTC2, DF66, DF68 and DF71 are shown in Table 1. These data show the followings. The rate of oxygen consumption, $\mathrm{J}_{0}$, of DF66 and DF71 were similar while $\mathrm{J}_{0}$ of DF68 was significantly reduced compared to that of FTC2. The $\mathrm{J}_{0}^{\mathrm{TET}}$ of the FLAG tagged-subunit 8 variants were not different from that of the wild-type version strain FTC2. The $\mathrm{J}_{0}{ }^{\mathrm{CCP}}$ of strains DF66 and DF68 was similar to that of the FTC2, while the $\mathrm{J}_{0}^{\text {CCCP }}$ of strain DF71 was found to be higher. The RCR of DF66 and DF68 were lower compared to that of the wild-type FTC2. The RCR of strain DF71 was similar to that of the FTC2. The MRC of the variants were similar to that of the FTC2. The $\mathrm{J}_{0}$ and RCR of DF68 were significantly lower compared to that of the FTC2.

Mitochondrial Respiration Parameters of Strains Expressing Wild-type and FLAG Tagged-subunit 8 Variants. The mitochondrial respiration parameters of strains FTC2, DF66, and DF68 are shown in Table 2. These data show the followings. The state 3 respiration rate of strain DF68 was significantly reduced. Similarly, state 3 respiration of strain DF66 was reduced compared to that of FTC2. While state 4 respiration rate of DF68 was reduced, the corresponding value of DF66 was not altered compared to that of the wild-type FTC2. The RCR of DF68 was reduced while that of DF66 was maintained. The maximal respiration rate of DF68 was lower compared to that of the wild-type, while that of DF66 was found to be higher.
Table 2 Mitochondrial respiration parameters of strains FTC2, DF66, and Df68

\begin{tabular}{cccc}
\hline Strains & \multicolumn{3}{c}{ Mitochondrail respiration parameters } \\
& $\begin{array}{c}\text { State 4(a) } \\
\left(\text { nmol O min }^{-1} \text { mg mitochondrial protein }^{-1}\right)\end{array}$ & $\begin{array}{c}\text { RCR } \\
\text { (state 3/state 4a) }\end{array}$ \\
\hline FTC2 & $130 \pm 9$ & $304 \pm 23$ & $2.3 \pm 0.1$ \\
DF66 & $111 \pm 7$ & $342 \pm 20$ & $2.2 \pm 0.1$ \\
DF68 & $80 \pm 13$ & $120 \pm 3$ & $1.5 \pm 0.2$ \\
\hline
\end{tabular}

State 4(a): state 4 respiration (absence of phosphorylation) prior to addition of ADP to induce state 3 respiration; State 3: a state of respiration at maximal phosphorylation rate; RCR: respiratory control ratio, is defined as state 3 respiration over state 4 (a) respiration.

b

\begin{tabular}{|c|c|c|c|}
\hline \multirow[t]{2}{*}{ Strains } & \multicolumn{3}{|c|}{ Mitochondrail respiration parameters } \\
\hline & $\begin{array}{r}\text { State } 4(\mathrm{~b}) \\
\left(\mathrm{nmol} \mathrm{O} \mathrm{min}^{-}\right.\end{array}$ & $\begin{array}{l}\text { Uncoupled Rate } \\
\text { nitochondrial } \text { protein }^{-1} \text { ) }\end{array}$ & $\begin{array}{c}\text { MRC } \\
\text { (uncoupled } \\
\text { rate/state 4b) }\end{array}$ \\
\hline FTC2 & $109 \pm 5$ & $314 \pm 13$ & $2.9 \pm 0.1$ \\
\hline DF66 & $106 \pm 9$ & $372 \pm 19$ & $3.5 \pm 0.1$ \\
\hline DF68 & $65 \pm 3$ & $194 \pm 3$ & $3.0 \pm 0.2$ \\
\hline
\end{tabular}

State 4(b): state 4 respiration (absence of phosphorylation) prior to addition of CCCP to induced uncoupled respiration; Uncoupled respiration is a state in which the respiratory chain is not kinetically controlled by the electrochemical proton gradient and therefore is dependent only on the activity of the respiratory chain; MRC: maximal respiratory control, is defined as uncoupled respiration over state 4(b) respiration.

Higher maximal respiration rate observed in strain DF66 resulted in the higher MRC of this strain. By contrast, the MRC of strain DF68 was not altered.

\section{DISCUSSION}

The present study mainly concerns the bioenergetic analysis of FLAG tagged-subunit 8 variants in order to verify the bioenergetic consequences of adjacent charged residues within the central hydrophobic domain (CHD) in combination with the presence of charged hexapeptide FLAG tag at the C-terminus. Since all of the strains tested bear FLAG epitope tag, it was therefore concluded that the aberrant functional performances displayed by the variants are likely mainly caused by the introduction of the charged residues within the CHD. These functional consequences may well be related to the fact that the presence of the charged residues within the CHD drastically reduces the hydrophobicity of the transmembrane region. In addition, the presence of FLAG epitope tag on the wild-type subunit 8 has been shown not to cause functional impairment on mitochondrial ATP synthase (Artika 2010).

The bioenergetic parameters of FLAG tagged-subunit 8 variants were analyzed at both cellular and mitochondrial

Table 1 Cellular respiration parameters of strains FTC2, DF66, DF68, and DF71

\begin{tabular}{|c|c|c|c|c|c|}
\hline \multicolumn{6}{|c|}{ Cellular respiration parameters } \\
\hline \multirow[t]{2}{*}{ Strains } & $\mathrm{J}_{0}$ & $\mathrm{~J}_{0}^{\mathrm{TET}}$ & $\mathrm{J}_{0}{ }^{\mathrm{CCCP}}$ & $\begin{array}{c}\mathrm{RCR} \\
\left(\mathrm{J}_{0} / \mathrm{J}_{0}^{\mathrm{TET}}\right)\end{array}$ & $\begin{array}{c}\mathrm{MRC} \\
\left(\mathrm{J}_{0}^{\mathrm{CCCP}} / \mathrm{J}_{0}^{\mathrm{TET}}\right)\end{array}$ \\
\hline & \multicolumn{3}{|c|}{$\left(\mathrm{nmol} \mathrm{O} \mathrm{min}^{-1} \mathrm{mg}\right.$ dry-cell-weight ${ }^{-1}$ ) } & & \\
\hline FTC2 & $21.7 \pm 0.1$ & $10.3 \pm 0.7$ & $27.9 \pm 3.1$ & $2.1 \pm 0.1$ & $2.7 \pm 0.2$ \\
\hline DF66 & $17.2 \pm 2.5$ & $10.1 \pm 1.8$ & $30.4 \pm 1.6$ & $1.7 \pm 0.1$ & $3.1 \pm 0.5$ \\
\hline DF68 & $13.9 \pm 0.9$ & $8.4 \pm 0.9$ & $26.6 \pm 2.1$ & $1.7 \pm 0.1$ & $3.2 \pm 0.6$ \\
\hline DF71 & $23.3 \pm 2.1$ & $10.9 \pm 1.9$ & $37.8 \pm 2.5$ & $2.2 \pm 0.2$ & $3.4 \pm 0.5$ \\
\hline
\end{tabular}


levels. Measuring bioenergetic parameters at the cellular level has been found to be a convenient preliminary test of mitochondrial performance. In addition, this procedure also allows the assessment of the bioenergetic characteristics of mutant strains having functional mitochondrial ATP synthase deficiencies from which good mitochondria may be difficult to isolate in sufficient amount, thus precluding bioenergetic studies on isolated mitochondria. Bioenergetic measurements made at cellular level also exclude functional alterations caused by artifacts during mitochondrial preparation (Law et al. 1995).

The data of cellular bioenergetic analysis of strains FTC2, DF66, DF68, and DF71 (Table 1) showed that the rate of oxygen consumption, $\mathrm{J}_{0}$, of DF66 and DF71 were similar while $\mathrm{J}_{0}$ of DF68 was significantly reduced compared to that of FTC2. $\mathrm{J}_{0}$ is defined as the physiological respiration rate, intermediate between state 3 and state 4 respiration. As the respiration rate reflects the activity of the mitochondrial ATP synthase, these data suggest that the functional coupling between the $F_{0}$ and $F_{1}$ sectors in DF68 is reduced as a consequence of the presence of adjacent negatively charged aspartate residues in the middle of the CHD. Generally these results are in agreement with the previously reported basic growth characteristics of the FLAG tagged-subunit 8 variants (Artika 2007) in that strain DF68 displayed the slowest growth rate. The generation times of FTC2, DF66, DF68, and DF71 were 6.9, 8.2, 11.1, and 7.2 hour respectively (Artika 2007). The generation time reflects the performance of the corresponding subunit 8 in the enzyme complex.

The respiration rate of the FLAG tagged-subunit 8 variants was also measured in the present of TET. The oxygen consumption rate $\left(\mathrm{J}_{0}{ }^{\mathrm{TET}}\right)$ under these conditions reflects the permeability of the inner mitochondrial membrane. $\mathrm{J}_{0}^{\mathrm{TET}}$ is defined as the inhibited rate or the apparent state 4 rate of respiration. The $\mathrm{J}_{0}^{\text {TET }}$ of the FLAG taggedsubunit 8 were not different from that of the wild-type strain FTC2. This indicates that the physical integrity of the inner mitochondrial membrane is maintained in the variants strains.

In the presence of protonophore, $\mathrm{CCCP}$, the respiration rate $\left(\mathrm{J}_{0}{ }^{\mathrm{CCCP}}\right)$ reflects the maximal respiratory capacity of the mitochondria. The $\mathrm{J}_{0}{ }^{\mathrm{CCCP}}$ of strains DF66 and DF68 was similar to that of the wild-type, while the $\mathrm{J}_{0}{ }^{\text {CCCP }}$ of strain DF71 was found to be higher. The reason for high $\mathrm{J}_{0}{ }^{\mathrm{CCCP}}$ of DF71 is unclear. The RCR DF68 was lower compared to that of the wild-type FTC2. This was mainly due to lower absolute values of respiration rate $\left(\mathrm{J}_{0}\right)$ of the DF68 compared to that of FTC2. The RCR of strains DF66 and DF71 was similar to that of the FTC2 indicating that the activity of the mitochondrial ATP synthase of these variants is maintained. The MRC of the variants were similar to that of the wildtype. To this end, the most profound functional defect was observed in the strain DF68 carrying adjacent negatively charged residues in the middle of CHD. The $\mathrm{J}_{0}$ and RCR of Df68 were significantly lower compared to that of the
FTC2. This indicates that the efficiency of the utilization of the proton motive force for $F_{1}$ activity is reduced in this strain.

Data from measurement of the gross cellular respiration rate suggested that the activity of the mitochondrial ATP synthase in strains DF66 and DF68 is reduced while that of DF71 is maintained. To confirm these findings, respiration measurements were also carried out on isolated mitochondria of DF66 and Df68. To obtain intact (well coupled) mitochondria for bioenergetic analysis, mitochondria were prepared from strains DF66 and DF68 according to the procedure described by Law et al. (1995). In this procedure, the mitochondria are released from the cells by enzymatic digestion of the cell walls rather that using mechanical force to break cells open.

Strain DF66 expresses variant having aspartate substitutions at residue positions 16 and 17. Data from analysis at the mitochondrial level (Table 2) showed that state 3 respiration of DF66 is lower than that of FTC2. The RCR of DF66, however, was similar to that of FTC2. These results indicate that there is possible functional impairment conferred by the strain DF66 due to in efficient coupling between the $\mathrm{F}_{1}$ and the $\mathrm{F}_{0}$ sectors. The MRC of strain DF66 is well maintained. This result indicates that the introduction of double aspartate residues at position 16 and 17 does not have major consequences on the respiratory chain.

Consistent with the data obtained from the cellular respiration assays, it was observed that state 3 respiration rate of strain Df68 was significantly reduced. State 4 respiration, RCR, and uncoupled respiration rate of DF68 were also reduced compared to that of the wild-type FTC2 (Table 2). Since the addition of the FLAG tag on the wildtype subunit 8 does not cause functional impairment on mitochondrial ATP synthase, it is therefore concluded that the aberrant functional performances displayed by DF68 are likely mainly caused by the introduction of the charged residues in the middle the central hydrophobic domain (CHD). These functional consequences may well be related to the fact that the presence of the charged residues in the middle the CHD drastically reduces the hydrophobicity of the transmembrane region.

Significant bioenergetic defects observed on strain DF68 having double aspartate substitutions in the middle of the CHD indicates that the functional coupling between $\mathrm{F}_{0}$ and $\mathrm{F}_{1}$ sector of DF68 is compromised. The respiration rate $\left(\mathrm{J}_{0}\right)$ is reduced by around $33 \%$. This indicates that significant reduction of mitochondrial ATP synthase has occurred due to the introduction of Asp23, 24. These results indicate that the position of the charged residues within the CHD is critical whether they are functionally defective or not. These data led to a conclusion that the hydrophobic character of the central of the CHD is important for the function of the mitochondrial ATP synthase. The two aspartate residues in the CHD may reduce the proton flow through the $\mathrm{F}_{0}$ channel by diverting protons to go in different directions in the membrane. Another possibility is that the charged residues alter protein-protein interaction of the coupling subunits between $\mathrm{F}_{1}$ and $\mathrm{F}_{0}$ sectors. 


\section{ACKNOWLEDGEMENTS}

I am grateful to AusAID for sponsoring me during the course of this study. I would like to thank Rodney J Devenish and Phillip Nagley of the Department of Biochemistry and Molecular Biology, Monash University, Victoria, Australia, for guidance and provision of facilities.

\section{REFERENCES}

Artika IM. 2006. Allotopic expression of a gene encoding FLAG taggedsubunit 8 of yeast mitochondrial ATP synthase. Hayati J Biosci 13: $36-8$

Artika IM. 2007. Structural and functional analysis of FLAG taggedsubunit 8 of yeast Saccharomyces cerevisiae mitochondrial ATP synthase. Microbiol Indones 1:33-6.

Artika IM. 2009. Membrane topology of subunit 8 of yeast Saccharomyces cerevisiae mitochondrial ATP synthase. Microbiol Indones 3:37-41.

Artika IM. 2010. Bioenergetic consequences of FLAG tag addition to the C-terminus of subunit 8 of yeast Saccharomyces cerevisiae mitochondrial ATP synthase. Hayati J Biosci 17:151-4.

Devenish RJ, Papakonstantinou T, Galanis M, Law RHP, Linnane AW, Nagley P. 1992. Structure/function analysis of yeast mitochondrial ATP synthase subunit 8. Ann NY Acad Sci 671:403-14.

Fronzes R, Weimann T, Vaillier J, Velours J, Brethes D. 2006. The peripheral stalk participates in the yeast ATP synthase dimerization independently of e and $g$ subunits. Biochemistry 45:6715-23.

Gearing DP, McMullen GL, Nagley P. 1985. Chemical synthesis of a mitochondrial gene designed for expression in the yeast nucleus. Biochem Int 10:907-15.
Hong S, Pedersen PL. 2008. ATP synthase and the actions of inhibitors utilized to study its roles in human health, disease, and other scientific areas. Microbiol Mol Biol Rev 72:590-641.

Kolodziej PA, Young RA. 1991. Epitope tagging and protein surveillance. Meth Enzymol 194:508-19.

Law RHP, Manon S, Devenish RJ, Nagley P. 1995. ATP synthase from Saccharomyces cerevisiae. Meth Enzymol 260:133-63.

Macreadie IG, Novitski CE, Maxwell RJ, John U, Ooi B, McMullen G, Lukins HB, Linnane AW, Nagley P. 1983. Biogenesis of mitochondria: the mitochondrial gene (aapl) coding for mitochondrial ATPase subunit 8 in Saccharomyces cerevisiae. Nucl Acids Res 11:4435-51

Nagley P, Farrell LB, Gearing DP, Nero D, Meltzer S, Devenish RJ. 1988. Assembly of functional proton-translocating ATPase complex in yeast mitochondria with cytoplasmically synthesised subunit 8 , a polypeptide normally encoded within the organelle. Proc Natl Acad Sci USA 85:2091-5.

Schafer K, Braun T. 1995. Monoclonal anti-FLAG antibodies react with a new isoform of rat $\mathrm{Mg}^{2+}$ dependent protein phosphatase . Biochem Biophys Res Commun 207:708-14.

Stephens AN, Khan MA, Roucou X, Nagley P, Devenish RJ. 2003. The molecular neighborhood of subunit 8 of yeast mitochondrial $F_{1} F_{0}-A T P$ synthase probed by cysteine scanning mutagenesis and chemical modification. J Biol Chem 278:17867-75.

Stephens AN, Roucou X, Artika IM, Devenish RJ, Nagley P. 2000 Topology and proximity relationships of yeast mitochondrial ATP synthase subunit 8 determined by unique introduced cysteine residues. Eur J Biochem 267:6443-51.

Van Ballmoos C, Wiedenmann A, Dimroth P. 2009. Essentials for ATP synthesis by $\mathrm{F}_{1} \mathrm{~F}_{0}$-ATP synthase. Ann Rev Biochem 78:649-72. 